\title{
Efeito do revestimento na aceitabilidade de mini-cenouras
}

\section{Effects of coating on the acceptability of baby-carrots}

\author{
Joesse Maria de Assis Teixeira Kluge Pereira ${ }^{1}$; Valéria Paula Rodrigues Minim²*; \\ José Benício Paes Chaves ${ }^{3}$; Fábio Yamashita ${ }^{4}$
}

\section{Resumo}

A aparência de um produto influencia a opinião do consumidor na sua decisão de compra e conseqüente consumo. Assim, a vida de prateleira e aceitabilidade de cenouras minimamente processadas é influenciada pela descoloração ou esbranquiçamento superficial que se desenvolve no produto. Este trabalho objetivou avaliar a aceitabilidade sensorial, em relação à cor, de mini-cenouras. Raízes de cenouras da variedade Indiana-F1 foram processadas na Indústria Mr. Rabbit Farm Ltda e divididas em dois lotes, onde um seguiu o fluxograma de processamento da indústria e ao outro foi acrescentada a etapa de revestimento em solução aquosa de $2 \%$ de polipeptídeos. Os dois lotes foram armazenados em expositores verticais, sob duas temperaturas, $5^{\circ} \mathrm{C}$ e $10^{\circ} \mathrm{C}$, para posterior avaliação sensorial. Os testes de aceitação das 4 amostras de mini-cenouras foram realizados com 1, 5, 9, 13, 17, 21 e 25 dias de armazenamento. Em cada tempo, de 100 a 125 consumidores avaliaram as mini-cenouras utilizando escala hedônica de 9 pontos. As avaliações foram realizadas em três supermercados existentes em Viçosa. Com um dia de armazenamento, a separação espacial das amostras sugeriu existir três grupos, um formado por amostras de mini-cenouras sem revestimento a $5^{\circ} \mathrm{C}$ e revestidas a $10^{\circ} \mathrm{C}$ e os outros dois por mini cenouras revestidas a $5^{\circ} \mathrm{C}$ e não revestidas a $10^{\circ} \mathrm{C}$. A distribuição dos consumidores demonstrou que a aceitação foi bastante homogênea, indicando não haver definição de preferência dos consumidores por nenhuma amostra específica. Do quinto ao $25^{\circ}$ dia, a separação espacial das amostras sugeriu existir dois grupos distintos em relação à aceitabilidade, um formado por minicenouras revestidas e outro por não revestidas, independente da temperatura de armazenamento, onde o grupo formado por amostras e mini-cenouras revestidas apresentou melhor aceitação por um número maior de consumidores. Os resultados indicaram a eficiência do revestimento na manutenção da cor característica da cenoura.

Palavras-chave: Revestimento comestível, mini-cenoura, processamento mínimo, teste de aceitação, mapa de preferência interno

\begin{abstract}
The consumer's opinion of a product is influenced by its appearance. This, in turn, will influence the decision to purchase and eat the product. Equally, the shelf life and acceptability of carrots subjected to minimal processing is influenced by the fading or superficial blanching that develops in the product. The aim of this work was to evaluate the sensorial acceptability of mini-carrots in relation to the colour.
\end{abstract}

\footnotetext{
1 Doutoranda em Ciência e Tecnologia de Alimentos, Universidade Federal de Viçosa, Bolsista Fapemig, , CEP 36570-000; Viçosa-MG. E-mail: joesseteixeira@yahoo.com.br

2 Doutorado em Ciência dos Alimentos (UNICAMP), Professora, do Departamento de Ciência e Tecnologia de Alimentos, Universidade Federal de Viçosa, Viçosa-MG. E-mail: vprm@ufv.br

3 Ph.D. Ciência de Alimentos (University of Maryland - EUA), Professor, do Departamento de Tecnologia de Alimentos, Universidade Federal de Viçosa, Viçosa-MG. E-mail: jbchaves@ufv.br

4 Doutorado em Engenharia de Alimentos (UNICAMP), Professor, Universidade Estadual de Londrina, Londrina-Pr, Brasil. Email: fabioy@uel.com.br

* Autor para correspondência
} 
Carrots of the variety Indiana-F1 were processed in the premises of ' Mr. Rabbit Farm Ltd ' and divided in two lots, where one followed the normal flow of processing for the industry and to the other a coating stage was added, using an aqueous $2 \%$ solution of polypeptides. The two lots were stored in upright racks, using two temperatures, $5^{\circ} \mathrm{C}$ and $10^{\circ} \mathrm{C}$, for subsequent sensorial evaluation. The acceptability tests of the 4 samples of baby-carrots were conducted after 1, 5, 9, 13, 17, 21 and 25 days of storage. Each time, from 100 to 125 consumers evaluated the baby-carrots using a 9 point hedonic scale. The evaluations were accomplished in three existing supermarkets in Viçosa. After one day of storage, the spacial separation of the samples suggested the existence of three groups, one formed by samples of baby-carrots without coating at $5^{\circ} \mathrm{C}$ and coated at $10^{\circ} \mathrm{C}$, and the other two comprised baby-carrots coated at $5^{\circ} \mathrm{C}$ and not coated at $10^{\circ} \mathrm{C}$. The consumers' distribution demonstrated that the acceptance was quite homogeneous, indicating there was no definite preference of the consumers for any specific sample. On the 5th to the 25th day, the spacial separation of the samples suggested there were two different groups with relation to their acceptability, one for samples of coated baby-carrots and the other for non-coated, independently of storage temperature. The group formed by samples of coated babycarrots showed better acceptance to a larger number of consumers. The results indicated the efficiency of the coating in the maintenance of the characteristic colour of the carrot.

Key words: Edible coating, baby-carrot, minimum processing, acceptance test, map of internal preference.

\section{Introdução}

A aparência de um produto influencia a opinião do consumidor em relação a outros atributos, quanto à sua decisão de compra e conseqüente consumo. Nos últimos anos, as hortaliças têm sido apresentadas ao consumidor na forma minimamente processadas e têm conquistado espaço nos grandes supermercados.

Para Howard e Griffin (1993), a vida de prateleira e aceitabilidade de cenouras minimamente processadas é influenciada pela descoloração ou esbranquiçamento que se desenvolve na superfície do produto.

O mecanismo de esbranquiçamento envolve respostas físicas e fisiológicas às injúrias sofridas ao longo do processamento. $\mathrm{O}$ esbranquiçamento dos tecidos resulta da desidratação parcial da superfície sendo uma resposta de ordem física, inicialmente reversível (TATSUMI; WATADA; WERGIN, 1991) e/ou ativação do metabolismo de compostos fenólicos e síntese de lignina, que é uma resposta de ordem fisiológica, irreversível, a qual é responsável pela descoloração de laranja intenso para laranja pálido (CARRASCO; CISNEROS-ZEVALLOS, 2002; CISNEROS-ZEVALLOS; SALTVEIT; KROCHTA, 1995; BOLIN; HUXOLL, 1991).

Visando evitar ou minimizar a mudança de cor nas cenouras minimamente processadas, diferentes métodos de preservação têm sido testados, como a imersão do produto em temperaturas mais baixas, visando a inibição da lignificação; umidificação com agentes higroscópicos para reduzir a desidratação superficial (CARRASCO; CISNEROSZEVALLOS, 2002); tratamento com vapor d'água com 100 ppm de cloro, acondicionamento e armazenamento a $2^{\circ} \mathrm{C}$, para diminuir a atividade enzimática (HOWARD; GRIFFIN; LEE, 1994) e revestimento ou filme comestível de forma a diminuir o esbranquiçamento superficial (HOWARD; DEWI, 1995; AVENA-BUSTILLOS et al., 1993).

É sempre importante para o consumidor e indústria avaliar com freqüência a qualidade sensorial dos produtos existentes no mercado.

Ao avaliar as alterações dos principais atributos sensoriais de cenouras minimamente processadas em dois tipos de corte durante o armazenamento, Resende et al. (2004) observaram que, em ambos os cortes, houve redução dos escores para cor e aparência e que cenouras raladas têm melhor cor e aparência quando comparadas à cenoura em rodelas.

O presente trabalho teve o objetivo de avaliar a aceitabilidade sensorial, em relação à cor, de mini cenouras com e sem revestimento, armazenadas a 5 e $10^{\circ} \mathrm{C}$. 


\section{Material e Métodos}

Raízes de cenouras da variedade Indiana-F1 foram processadas na Indústria Mr. Rabbit Farm Ltda e divididas em dois lotes, onde um seguiu o fluxograma de processamento da indústria e ao outro foi acrescentada a etapa de revestimento em solução aquosa de $2 \%$ de polipeptídeos.

Os dois lotes foram armazenados em dois expositores verticais com ventilação forçada, sob duas temperaturas distintas, $5{ }^{\circ} \mathrm{C}$ e $10{ }^{\circ} \mathrm{C}$ para posterior avaliação sensorial.

\section{Teste de aceitação}

A avaliação da aceitabilidade sensorial do produto foi realizada em três supermercados de médio porte da cidade de Viçosa-MG, no período da tarde (entre 14:30 e 18:00 horas).
Os testes de aceitação das quatro amostras de mini cenouras (com revestimento $5{ }^{\circ} \mathrm{C}$, sem revestimento $5{ }^{\circ} \mathrm{C}$, com revestimento $10{ }^{\circ} \mathrm{C}$ e sem revestimento $10{ }^{\circ} \mathrm{C}$ ) foram realizados com $1,5,9,13,17,21$ e 25 dias de armazenamento. Em cada tempo, de 100 a 125 consumidores que estiveram no supermercado em cada dia e horário de avaliação, avaliaram as mini-cenouras.

As quatro amostras foram servidas em pratos descartáveis de fundo branco, e foram apresentadas e analisadas pelos consumidores em uma única sessão. Em cada tempo de análise experimental, os provadores avaliaram as amostras, utilizando a escala hedônica de nove pontos (Quadro 1), indicando o quanto gostaram ou desgostaram das amostras de mini-cenouras em relação à cor (esbranquiçamento da mini-cenoura).

\section{ESCALA HEDÔNICA}

Nome:

Data:

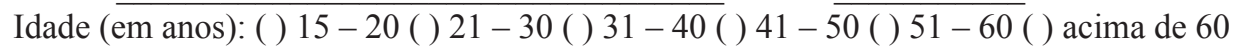

Por favor, avalie a amostra de cenoura minimamente processada, utilizando a escala baixo para descrever o quanto você gostou ou desgostou de cada atributo (cor e impressão global) do produto.

Código da amostra:

9 - gostei extremamente

8 - gostei muito

7 - gostei moderadamente

6 - gostei ligeiramente Cor:

5 - nem gostei nem desgostei

4 - desgostei ligeiramente

3 - desgostei moderadamente

2 - desgostei muito

1 - desgostei extremamente

Quadro 1. Ficha do teste de aceitação utilizada na avaliação da cor de mini cenouras 


\section{Mapa de preferência interno}

A análise estatística dos dados foi feita pela técnica de Mapa de Preferência Interno, na qual considera-se a resposta individual de cada consumidor e não apenas a média do grupo de consumidores que avaliaram o produto.

Para a obtenção do Mapa de Preferência Interno, os dados de aceitação foram organizados em uma matriz de amostras (em linhas) e consumidores (em colunas) e submetida à Análise de Componentes Principais (ACP) (MINIM, 2006).

Os dados obtidos na ACP foram dispostos em um gráfico de dispersão, representando as amostras (tratamentos) e os "loadings" (cargas), em relação aos dois primeiros componentes principais.

Foram empregados procedimentos do sistema estatístico SAS (STATISTICAL ANALYSIS SYSTEM - SAS (2003), versão 8.0, licenciada para a Universidade Federal de Viçosa, 2003.

\section{Resultados e Discussões}

Com os dados obtidos no teste de aceitação das quatro amostras de mini-cenouras, foi realizada a análise por Mapa de Preferência Interno, da cor, para cada tempo de armazenamento (Figura 1).

O Mapa de Preferência Interno gerou, em espaço multidimensional, as coordenadas relativas ao produto, as quais por sua vez, foram formadas de acordo com as respostas dos provadores. Os mapas da Figura 1 foram gerados por meio dos Componentes Principais (CP-Y1, CP-Y2) que, juntos, explicaram de 76 a $89 \%$ da variação entre as amostras quanto à sua aceitação e o CP-Y1 explicou de 44,3 a 73,8 da variação em relação à cor durante o tempo de armazenamento.
$\mathrm{Na}$ Figura 1, cada ponto em preto representa as correlações entre os dados de aceitação de um consumidor e os dois primeiros componentes principais. Dessa forma, cada ponto preto está associado a um provador.

Verificou-se que, com um dia de armazenamento, a separação espacial das amostras sugeriu a existência de três grupos, um formado por amostras de minicenouras sem revestimento a $5{ }^{\circ} \mathrm{C}$ e revestidas a $10^{\circ} \mathrm{C}$ e os outros dois por mini-cenouras revestidas a $5{ }^{\circ} \mathrm{C}$ e não revestidas a $10{ }^{\circ} \mathrm{C}$ (Figura 2-A).

A distribuição dos consumidores demonstrou que a aceitação foi bastante homogênea, indicando que não houve definição de preferência dos consumidores por nenhuma amostra específica.

\section{Conclusões}

A aplicação do revestimento comestível à base de $2 \%$ de polipeptídeos favoreceu a manutenção da qualidade sensorial avaliada das mini cenouras, a cor, para as duas temperaturas.

Os resultados indicaram que, independente da temperatura de armazenamento $\left(5\right.$ ou $10^{\circ} \mathrm{C}$ ), o revestimento avaliado foi eficiente na manutenção da cor característica da cenoura.

\section{Agradecimentos}

Indústria Mr. Rabbit Farm Ltda., por ceder e processar todo o material vegetal utilizado nesta pesquisa. 


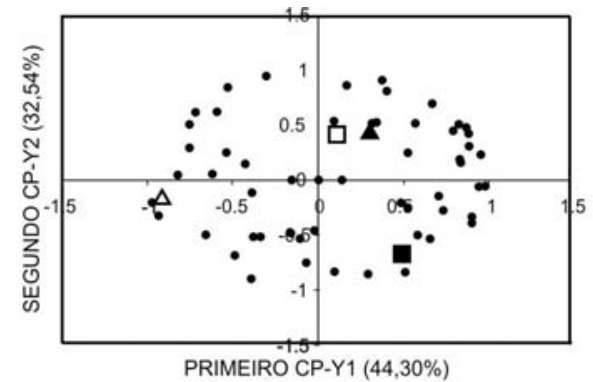

A
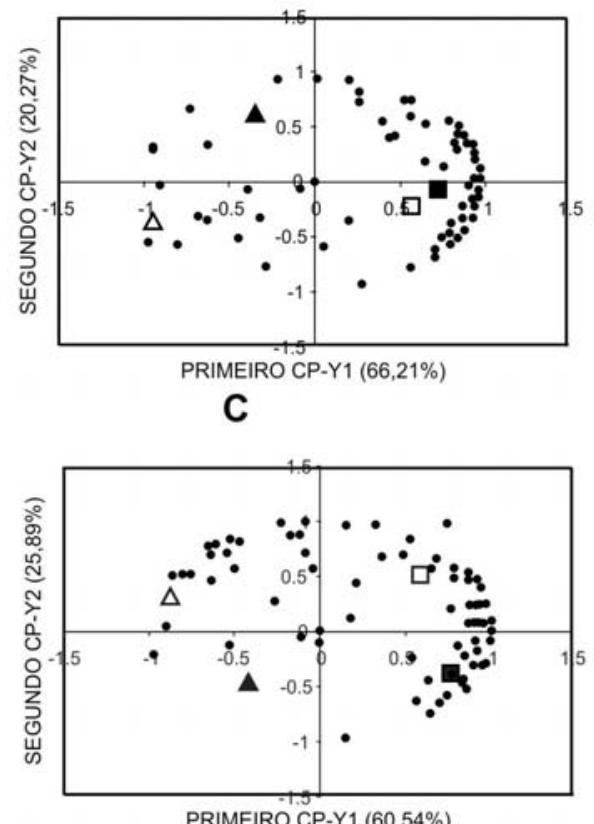

E

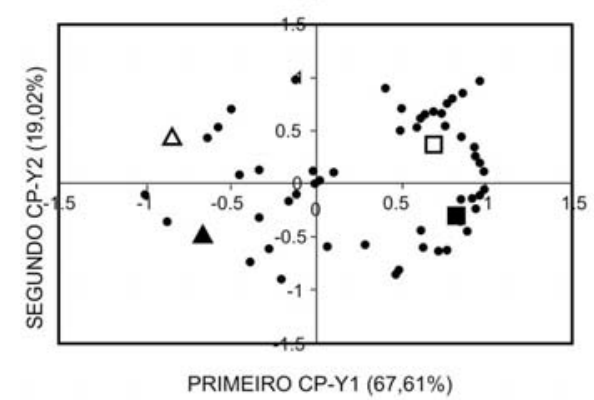

G

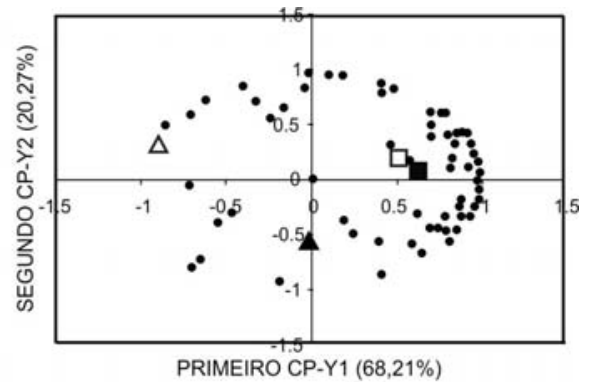

B
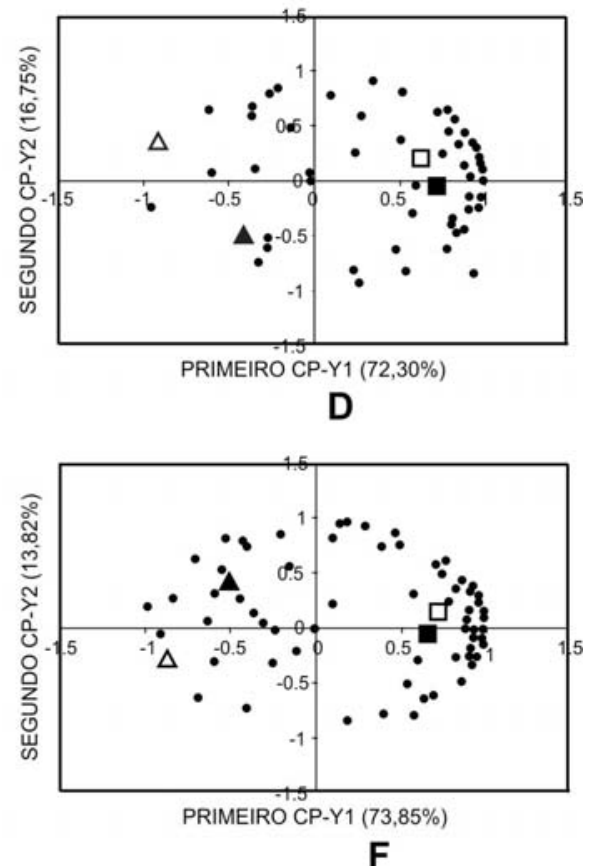

$\mathbf{F}$

Não revestida $5^{\circ} \mathrm{C}$

Revestida $5^{\circ} \mathrm{C}$

$\triangle$ Não revestida $10^{\circ} \mathrm{C}$

Revestida $10^{\circ} \mathrm{C}$

Consumidores

Figura 1. Mapa de Preferência Interno, do atributo cor, para mini cenouras revestidas e não revestidas nos diferentes tempos de armazenagem: Gráficos A (1 dia), B (5 dias), C (9 dias), D (13 dias), E (17 dias), F (21 dias) e G (25 dias). 


\section{Referências}

AVENA-BUSTILLOS, R. J.; CISNEROS-ZEVALLOS, L. A.; KROCHTA, J. M.; SALTVEIT, M. E. Optimization of edible coatings on minimally processed carrots using response surface methodology. Transactions of the ASAE, Saint Joseph, v. 36, n. 3, p. 801-805 1993.

BOLIN, H. R.; HUXSOLL, C. C. Control of minimally processed carrot (Daucus carota) surface discoloration caused by abrasion peeling. Journal of Food Science, Chicago, v. 56, n. 2, p. 416-418, 1991.

CARRASCO, E. U.; CISNEROS-ZEVALLOS, L. Efecto del escaldado y recubrimiento higroscópico sobre la calidad de zanahorias (Daucus carota var Chantenay) pre-cortadas durante el almacenamiento. Archivos Latinoamericanos de Nutrición, Caracas, v. 52, n. 2, p. 187-192, 2002.

CISNEROS-ZEVALLOS, L.; SALTVEIT, M. E.; KROCHTA, J. M. Mechanism of surface white discoloration of peeled (minimally processed) carrots during storage. Journal of Food Science, Chicago, v. 60, n. 2, p. 320-33, 1995.

HOWARD, L. R.; GRIFFIN, L. E. Lignin formation and surface discoloration of minimally processed carrot sticks. Journal of Food Science, Chicago, v. 58, n. 5, p. 1065-1067, 1993.
HOWARD, L. R.; GRIFFIN, L. E.; LEE, Y. Steam treatment of minimally processed carrot sticks to control surface discoloration. Journal of Food Science, Chicago, v. 59, n. 2, p. 356-58, 1994.

HOWARD, L. R.; DEWI, T. Sensory, microbiological and chemical quality of mini peeled carrots as affected by edible coating treatment. Journal of Food Science, Chicago, v. 60, n. 1, p. 142-144, 1995.

MINIM, V. P. R. Análise sensorial: estudo com consumidores.Viçosa: Ed. da UFV, 2006. 225p.

RESENDE, J.M.; COELHO, A.F.S.; CASTRO,, E. C.; SAGGIN JÚNIOR, O.J.; NASCIMENTO,, T.; BENEDETTI, B. C. Modificações sensoriais em cenoura minimamente processada e armazenada sob refrigeração. Horticultura Brasileira, Brasília, v. 22, n.1, p. 147-150, jan-mar, 2004.

STATISTICAL ANLYSIS SYSTEM - SAS. Institute Inc., North Carolina, USA. Versão 8.0, licenciada para Universidade Federal de Viçosa. Cary: SAS Institute, 2003.

TATSUMI, Y.; WATADA, A. E.; WERGIN, W. P. Scanning electron microscoscopy of carrot stick surface to determine cause of white translucent appearance. Journal of Food Science, Chicago, v. 56, n. 5, p. 13571364, 1991. 